\title{
(2) OPEN ACCESS \\ Effect of valproic acid on functional bleb morphology in a rabbit model of minimally invasive surgery
}

\author{
Zhu Li Yap (1) , ' Li-Fong Seet, ${ }^{1}$ Stephanie WL Chu, ${ }^{2}$ Li Zhen Toh, ${ }^{2}$ \\ Farah Ilyana Ibrahim, ${ }^{3}$ Tina T Wong (1) ${ }^{1}$
}

${ }^{1}$ Department of Ophthalmology, Singapore Eye Research Institute, Singapore ${ }^{2}$ Ocular Therapeutics \& Drug Delivery, Singapore Eye Research Institute, Singapore ${ }^{3}$ Training and Education, Singapore National Eye Centre, Singapore

\section{Correspondence to}

Dr Li-Fong Seet, Department of Ophthalmology, Singapore Eye Research Institute, Singapore; seet.li.fong@seri.com.sg

ZLY and L-FS contributed equally.

Received 20 December 2020 Accepted 19 June 2021

\section{ABSTRACT}

Purpose To determine the effect of valproic acid (VPA) on bleb morphology and scar characteristics in a rabbit model of minimally invasive glaucoma surgery (MIGS).

Methods Nine New Zealand white rabbits were subjected to MIGS with intraoperative implantation of the PreserFlo MicroShunt. Rabbits were then administered with subconjunctival injections of phosphate buffered saline (PBS) $(n=4)$ or with VPA $(n=5)$. Bleb morphology was examined by slit-lamp biomicroscopy and in vivo confocal microscopy. Postoperative day 28 tissues were examined by immunohistochemical evaluation and label-free multiphoton microscopy to visualise the collagen matrix, by terminal deoxynucleotidyl transferase dUTP nick-end labelling assay and immunofluorescent labelling for Ki67 expression to detect apoptosis and cell growth, and by real-time quantitative PCR to measure Col1a1, Fn, and Smad6 transcript expression.

Results VPA-treated blebs were detectable on day 28 , while the PBS-treated blebs were not detectable by day 14 . VPA-treated blebs were diffuse, extended posteriorly with near normal conjunctival vascularity and featured a combination of reticular/blurred stromal pattern with evidence of relatively large stromal cysts. Instead of the deposition of thick, disorganised collagen fibres characteristic of the PBS bleb, the VPA bleb contained conspicuously thinner collagen fibres which were associated with similarly thinner fibronectin fibres. In corroboration, Col1a1 and Fn mRNA expression was reduced in the VPA blebs, while increased Smad6 expression implicated the disruption of the transforming growth factor beta pathway. Apoptosis and cell growth profiles appeared similar with both treatments.

Conclusions The results support the application of VPA to enhance bleb morphology associated with good bleb function in MIGS with no apparent cytotoxicity.

\section{INTRODUCTION}

Glaucoma is an irreversible optic neuropathy and a major cause of blindness globally. Elevated intraocular pressure (IOP) is the main risk factor for glaucoma, and reducing IOP is the only known effective modifiable factor in managing and slowing its progression. ${ }^{1}$ Treatment may include topical medications, lasers or glaucoma filtration surgery (GFS). GFS is generally considered the gold standard for providing the most reliable IOP reduction. Conventional GFS is an incisional procedure where a small part of the trabecular meshwork and/or adjacent corneoscleral tissue is removed to provide a drainage route for aqueous humour to exit underneath the conjunctiva. GFS include the recently introduced microinvasive or minimally invasive glaucoma surgery (MIGS), which as the name implies, is considered to be less invasive. ${ }^{23}$ MIGS includes the use of ab externo devices to create an outflow shunt for the aqueous humour from the anterior chamber (AC) into the subconjunctival space. As the subconjunctival space is inherently prone to fibrosis, a major obstacle to GFS and ab externo MIGs is therefore the high risk of scarring which leads to failure of these procedures.

The formation of scars, composed mainly of disorganised collagen, not only blocks the ability of the surgically created fistula to drain fluid from the AC but also may disturb conjunctival architecture, which in turn disrupt normal blood and lymphatic vasculatures necessary for fluid drainage. ${ }^{4}$ To control scarring, adjunct agents that reduce the amount of fibrotic collagen deposited are routinely administered intraoperatively. Mitomycin C (MMC) is the most commonly used antifibrotic adjunct for both conventional surgeries and MIGS. ${ }^{5}$ Although MMC is highly effective in reducing the postoperative scarring response, surgeries continue to fail. A 2-year follow-up study indicated a failure rate of 43.4\% in American-Asian patients who had undergone intraoperative treatment with MMC. ${ }^{6}$ Moreover, MMC is associated with serious adverse effects by causing non-specific cytotoxicity, leading to the development of thin, avascular blebs that are prone to hypotony, blebitis and endophthalmitis. ${ }^{78}$ A safer and more effective antifibrotic therapy is therefore an unmet need for GFS and MIGS. ${ }^{8}$

Valproic acid (VPA) is a small molecule drug widely used for the treatment of neurological disorders, with potential efficacy for numerous diseases. ${ }^{9}$ In particular, VPA has been recognised as potential antifibrotic therapy. ${ }^{10}$ We have also demonstrated that VPA was effective in reducing collagen production in the mouse model of conjunctival scarring via upregulation of Smad6 in vivo. ${ }^{11}$ In this study, we evaluated the capacity of VPA to modulate collagen morphology in a rabbit model of MIGS based on the PreserFlo MicroShunt. ${ }^{12}$ This is an ab externo implant that promises to bridge the gap between trabecular bypass/ablative procedures and traditional GFS. The PreserFlo creates a subconjunctival bleb, similar to GFS, and, given the requirement for extensive conjunctival dissection, is subject to the risk of subconjunctival fibrosis. We reveal that VPA treatment promoted a bleb appearance associated with good filtration function as well as modulated 
the content and organisation of the collagen matrix in the postoperative scar by preventing the deposition of thick and disorganised collagen fibres. These findings therefore support the application of VPA as an effective antifibrotic agent for $a b$ externo MIGS.

\section{MATERIALS AND METHODS Rabbit model of MIGS}

All animal experiments were approved by the Institutional Animal Care and Use Committee and treated in accordance with the Association for Research in Vision and Ophthalmology Statement on the Use of Animals in Ophthalmic and Vision Research. New Zealand White (NZW) rabbits (2.0-2.4 kg), 12-14 weeks old, were acclimatised for 7 days before surgery at the SingHealth Experimental Medical Centre (Singapore General Hospital, Singapore). The rabbits were anaesthetised with a combination of ketamine (Ketaset; Fort Dodge Animal Health, Southampton, UK) and medetomidine $\mathrm{HCl}$ (Domitor; Pfizer Animal Health, Sandwich, UK). A fornix-based conjunctival flap was raised and blunt dissection of the subconjunctival space was performed approximately $3 \mathrm{~mm}$ along the limbus and $5 \mathrm{~mm}$ posteriorly. The AC was deepened by injecting $0.2 \mathrm{~mL}$ of basal salt solution on a $30 \mathrm{G}$ needle, and the PreserFlo implant (Santen, Japan) was then inserted as per manufacturer's instructions. PreserFlo MicroShunt, manufactured and provided by InnFocus, is an implantable glaucoma drainage device made of a flexible poly(styrene-block-isobutylene-block-styrene) polymer with a tube of $350 \mu \mathrm{m}$ outer diameter and a lumen of $70 \mu \mathrm{m} .{ }^{12}$ It has triangular fins that prevent migration of the tube into the AC. The device is designed to be implanted under the subconjunctival/Tenon space. A $25 \mathrm{G}, 1 \mathrm{~mm}$ blade created the start of a sclerostomy $2.5 \mathrm{~mm}$ behind the limbus, followed by a 25 $G$ needle which was used to create a tunnel entry into the AC and completing the sclerostomy. The implant was then placed with $2.5 \mathrm{~mm}$ in the $\mathrm{AC}$ and $3 \mathrm{~mm}$ sitting on the sclera within the conjunctival pocket. Watertight closure of the conjunctival incision was performed using 10-0 nylon via purse-string sutures and, where necessary, a mattress suture. Only the left eye of each rabbit was operated on, and the surgical procedure was performed at the same site superiorly in each animal. One drop each of guttae chloramphenicol and Betnesol-N (Glaxo Wellcome, Uxbridge, UK) ointment was instilled at the end of surgery. The operated eyes were treated with $1 \times$ subconjunctival injections of $0.1 \mathrm{~mL}$ volume of phosphate buffered saline (PBS) or $300 \mu \mathrm{g} / \mathrm{mL}$ VPA at the following postoperative time points: day $0,1,2,3,4,5,6,7,10,14$ and 21 . Topical antibiotic and steroid drops were administered daily in both groups for 3 weeks postoperatively. The animals were sacrificed on day 28 and eyes were enucleated for further analyses (PBS, $n=4$; VPA, $n=5$ ).

\section{Primary rabbit conjunctival fibroblast cell culture}

Primary conjunctival fibroblasts were obtained from the eyes of NZW rabbits and cultured as described previously. ${ }^{13}$ Treatment with increasing concentrations of VPA (Sigma-Aldrich Co., St. Louis, Missouri, USA) was carried out for 72 hours for real-time quantitative PCR analysis and for 5 days for real-time cell proliferation evaluation.

\section{Real-time quantitative PCR (qPCR)}

For mRNA analyses of the rabbit model, a small portion of the operated conjunctiva was excised on day of sacrifice (day 28). A similar area of unoperated conjunctiva in the contralateral eye of each rabbit was harvested to obtain baseline value for calculation of fold change in transcript expression. Both rabbit conjunctival tissues and fibroblasts were processed and analysed by qPCR as described previously. ${ }^{14}$ Samples were collected in RNAlater solution (Life Technologies, Carlsbad, California, USA) and lysed using the Vibra-Cell ultrasonic processor VCX130 (Sonics \& Materials, Connecticut, USA). Total RNA was recovered with Trizol Reagent (Invitrogen Corp, California, USA) and the RNeasy kit (Qiagen, Valencia, California, USA) as described previously. ${ }^{14}$ Total RNA was reverse-transcribed into cDNA using random hexamer primers (Invitrogen Corp) with Superscript III reverse transcriptase (Invitrogen Corp). All qPCR reactions comprising the Power SYBR Green PCR Master Mix (Applied BioSystems, California, USA) were performed in triplicate in volumes of $10 \mu \mathrm{L}$ in 384-well microtitre plates and run using the Roche LightCycler 480 System (Roche Diagnostics Corp, Indianapolis, USA). All mRNA levels were measured as CT threshold levels. Rna18s1 was determined to be the most suitable housekeeping gene of four analysed (Actb, Rna18s1, Gapdh and Rpl13a) using the NormFinder software. ${ }^{15}$ Value for each operated eye was calculated as fold change relative to the corresponding contralateral unoperated eye by the $2^{-\Delta \Delta \mathrm{CT}}$ method. Primers used were Rna18s1-forward, 5'-CTTTGGTCGCTCGCTCCTCTCC3', and Rna18s1-reverse, 5'-TCTGATAAATGCACGCATCC CACAC-3'; Col1a1-forward, 5'-CGATGGCTTCCAGTTCGAGT-3'; Col1a1-reverse, 5'-CTACGCTGTTCTTGCAGTG-3'; Fn1-forward, 5'-GGATGTTCCCTCCACAGTTCA-3', and Fn1reverse, 5'-TGGTCCGCCTAAAACCATGT-3'; Smad6-forward, 5'-ACCAACTCCCTCATCACTGC-3', and Smad6-reverse, 5'- GTCGTACACGGCGTAGAGG-3'.

\section{Real-time cell proliferation analysis}

The xCelligence real time cell analyser (Roche Diagnostics $\mathrm{GmbH}$, Penzberg, Germany) was used to assess cell proliferation according to manufacturer's instructions. Fibroblasts were seeded onto the E-Plate 96 (Roche Diagnostics $\mathrm{GmbH}$ ) wells at 1000 cells/well in normal culture medium, in quadruplicate. Adherent cell growth/density was monitored continuously for up to 5 days.

\section{Live imaging of rabbit eyes}

Postoperative observations were performed at 2 and 4 weeks before sacrifice. Rabbits were anaesthetised before imaging was performed. Slit-lamp microscopy was performed using Righton LED slit-lamp MW50D (Right Mfg Co, Japan). In vivo confocal microscopic examinations of blebs were performed using Hrt3 microscope (Heidelberg Engineering, Heidelberg, Germany).

\section{Immunohistochemical evaluation of cryosections}

During enucleation, the upper lid was removed together with the whole eye to preserve the bleb and superior conjunctiva. Each tissue sample was fixed with paraformaldehyde and then placed in a slurry of optimal cutting temperature compound in cryomold before freezing in dry ice and storage in a $-80^{\circ} \mathrm{C}$ freezer until ready for sectioning using the MicromHM550 (Carl Zeissd). Cryosections $(5 \mu \mathrm{m})$ were then subjected to histochemical or immunofluorescent analyses. Histochemical evaluation of operated conjunctival cryosections of the bleb area was performed by staining with $\mathrm{H} \& \mathrm{E}$ or picrosirius red as described previously. ${ }^{14}$

The Masson trichrome stain kit was purchased from Electron Microscopy Sciences (Hatfield, Pennsylvania, USA) and used according to the manufacturer's instructions. Polarisation microscopy for picrosirius red-stained cryosections was performed 
using the Nikon Eclipse Ti microscope (Nikon Instruments, New York, USA). Immunostaining for Col1a1 and fibronectin (FN) were performed using antibodies from Abnova Corp. (Littleton, Colorado, USA) and Abcam plc (Cambridge, UK), respectively. Labelling by primary antibodies was detected using secondary antibodies conjugated to Alexa Fluor-594 (red fluorescence) or Alexa Fluor-488 (green fluorescence), both obtained from Invitrogen Corp. (Thermo Fisher Scientific, Waltham, Massachusetts, USA). Nuclei were visualised by mounting the cells in DAPI (4', 6-diamidino-2-phenylindole)-containing Vectashield mounting medium (Vector Laboratories, California, USA). Labelled tissues were visualised using the Nikon Eclipse Ti confocal microscope (Nikon Instruments).

\section{Terminal deoxynucleotidyl transferase dUTP nick-end labelling (TUNEL) assay and costaining with Ki67 on cryosections}

End labelling of exposed 3'-OH ends of DNA fragments with fluorescein-12-dUTP on cryosections was performed using the DeadEnd Fluorometric TUNEL System according to the manufacturer's instructions (Promega, Madison, USA). The TUNEL assay was combined with staining for Ki67 using antibody obtained from Abcam plc (Cambridge, UK). Labelling by the Ki67 primary antibody was detected using goat anti-rabbit IgG secondary antibody conjugated to Alexa Fluor 568 (A-11011) obtained from Invitrogen Corp. (Thermo Fisher Scientific). Staining of the cell nucleus was achieved by mounting the cryosections in DAPI-containing Vectashield mounting medium (Vector Laboratories). Sections were visualised using the Nikon Eclipse Ti confocal microscope (Nikon Instruments).

\section{Multiphoton microscopy}

Images of unstained cryosections were acquired on a fully automated, programmable, multiphoton imaging platform (Genesis 200; HistoIndex Pte, Singapore). Laser excitation was carried out at $780 \mathrm{~nm}$, and forward-scatter two-photon excitation (TPE) and second harmonic generation (SHG) signals were detected simultaneously using dedicated photomultiplier tubes for each channel at 550 and $390 \mathrm{~nm}$, respectively, and by using a dichroic mirror (450DCLP, Omega) to separate TPE from SHG. Images were acquired at $20 \times$ with $512 \times 512$ pixel resolution with $2 \times$ frame averaging feature, and each image had a dimension of $200 \times 200 \mu \mathrm{m}^{2}$. TPE sensitivity was set to 0.75 , and SHG sensitivity to 0.65 . A bandpass filter with centre wavelength at $550 \mathrm{~nm}$ and bandwidth of $88 \mathrm{~nm}$ was set in front of the TPE photodetector. Laser baseline power was set at 0.45 . The laser beam with horizontal polarisation was used for excitation.

\section{Statistical analysis}

All data are expressed as mean \pm SD. Where only two treatment conditions were compared, the significance of differences between the two conditions was determined by the two-tailed Student t-test using Microsoft Excel V.5.0 software. Statistical significance was defined as a $\mathrm{p}$ value of $<0.05$.

\section{RESULTS}

\section{Effective VPA concentration for rabbit model of MIGS}

We have previously shown that mRNA expression of Col1a1 was effectively suppressed by VPA in primary mouse conjunctival fibroblasts and that the optimum dosage determined in vitro was also effective when used in vivo in the mouse model of conjunctival scarring. ${ }^{11}$ To determine the optimum dose of VPA for inhibition of Col1a1 expression in the rabbit, we performed
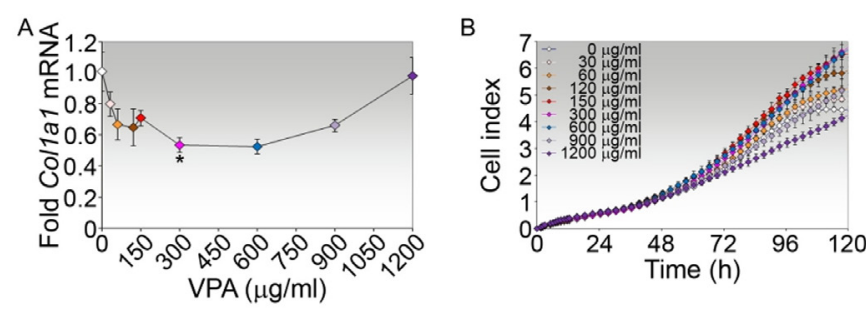

Figure 1 VPA dosage for inhibition of Col1a1 expression in primary rabbit conjunctival fibroblasts. (A) Real-time PCR analysis of Col1a1 mRNA in fibroblasts treated with increasing concentrations of VPA for 72 hours. Data are presented as mean fold change \pm SD relative to untreated cells. ${ }^{*} \mathrm{P}$ value comparing fold mRNA between treatment with $300 \mu \mathrm{g} / \mathrm{mL}$ VPA and untreated controls is shown. (B) Real-time cell proliferation analysis of fibroblasts treated with increasing concentrations of VPA. Data are presented as mean cell index $\pm S D$ of triplicates. VPA, valproic acid.

a similar titration study of VPA on primary cultures of rabbit conjunctival fibroblasts. Similar to the mouse, VPA at a concentration of $300 \mu \mathrm{g} / \mathrm{mL}$ (or $2 \mathrm{mM}$ ) significantly reduced Col1a1 mRNA expression by a mean of 1.88 -fold (figure $1 \mathrm{~A}$ ) without affecting real-time cell growth measured over a period of 5 days (figure 1B). Hence, subsequent investigation of VPA for antifibrotic activity in the rabbit model of MIGS was performed using VPA at $300300 \mu \mathrm{g} / \mathrm{mL}$.

\section{Bleb morphology by slit-lamp microscopy}

The rabbit model of MIGS was performed using the PreserFlo MicroShunt and treatment with VPA involved a total of 11 subconjunctival injections over the course of 3 weeks. A single unmasked observer evaluated the effect of VPA on tissue morphology at the operated site by slit-lamp microscopy. Blebs were recorded as being present or absent. Quantitative estimation of the bleb area was not performed as we considered it to be too inaccurate given that injections in both groups continued up to day 21. The characteristic tubular structure of the implant in the operated area may be observed from its position under the conjunctiva and Tenon's capsule (arrows, figure 2). The bleb, resulting from aqueous outflow from the AC into the subconjunctival space through the distal end of MicroShunt, may be observed as a diffused, elevated subconjunctival fluid pocket in the proximity of the implant (arrowheads, figure 2). In the tissue treated with PBS, there was no observable bleb by day 14 as the operated area appeared flat, suggesting that scarring had occurred as early as 2 weeks postsurgery (figure 2). On the other hand, an observable, posteriorly diffused, fluid-filled bleb may be detectable in the proximity of the implant in the VPA-treated eye for up to 28 days postsurgery (arrowheads, figure 2).

Notably, the area over the implant outlet in the day 28 PBStreated tissue was highly vascularised, in comparison with the visibly less vascularised bleb area in the VPA-treated eye (arrowhead, figure 2). In general, the blood vessels that traverse the VPA-treated bleb area were also finer than those in the PBStreated bleb. These observations suggest that VPA was effective in maintaining bleb survival and modulates the vascularisation in the rabbit model of MIGS.

\section{Bleb stroma organisation by confocal microscopy}

Confocal microscopy has been used to correlate bleb appearance and function. ${ }^{4}$ Significant positive correlating features for good bleb function in patients include large cyst size and reduced 


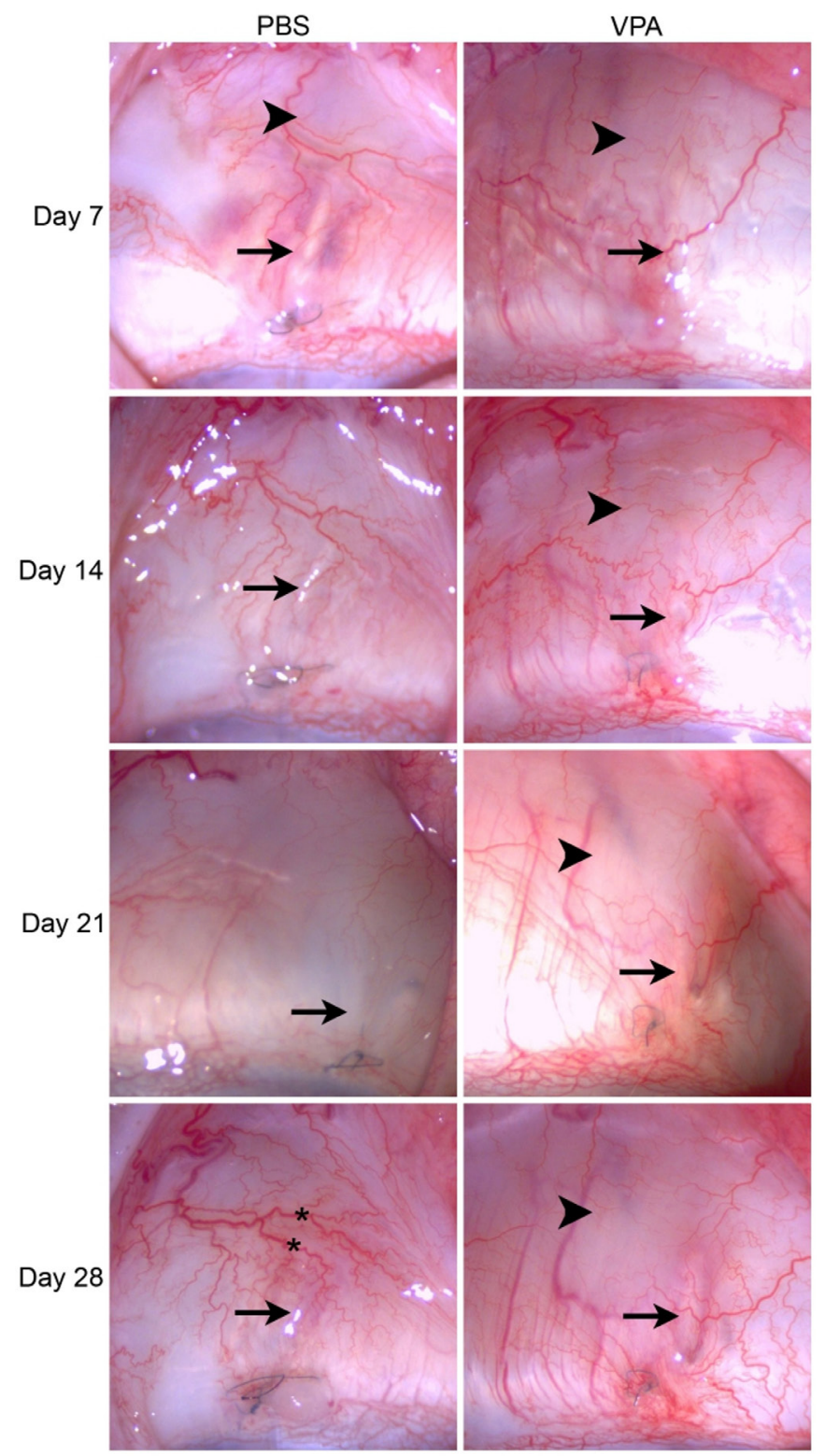

Figure 2 Slit-lamp biomicroscopy of the operated conjunctivas in the rabbit model of minimally invasive glaucoma surgery. Rabbits were treated by subconjunctival injections of PBS or $300 \mu \mathrm{g} / \mathrm{mL}$ VPA for up to 3 weeks. Eyes of the same rabbit eye injected with either PBS or VPA were imaged on a weekly basis up to day 28. The MicroShunt may be observed in the subconjunctival space overlying the sclera (arrows). Observable blebs are indicated by arrowheads. Prominent blood vessels may be observed crossing over the area near the distal end of the MicroShunt (asterisks). PBS, phosphate buffered saline; VPA, valproic acid.

vascular vessel density and tortuosity. We therefore examined the day 28 operated conjunctiva by confocal microscopy to determine the stromal organisation after treatment with VPA. The subconjunctival stromal matrix was profoundly distinct between the PBS-treated and VPA-treated eyes. The PBS-treated conjunctival stroma consisted of a compact and hyper-reflective pattern with densely packed fibres. Moreover, PBS treatment resulted in very small cysts being formed. In contrast, the VPAtreated matrix consisted of a combination of a reticular stromal pattern characterised by loose criss-crossed fibres (figure 3A) and a blurred stromal pattern (figure 3B). The VPA-treated

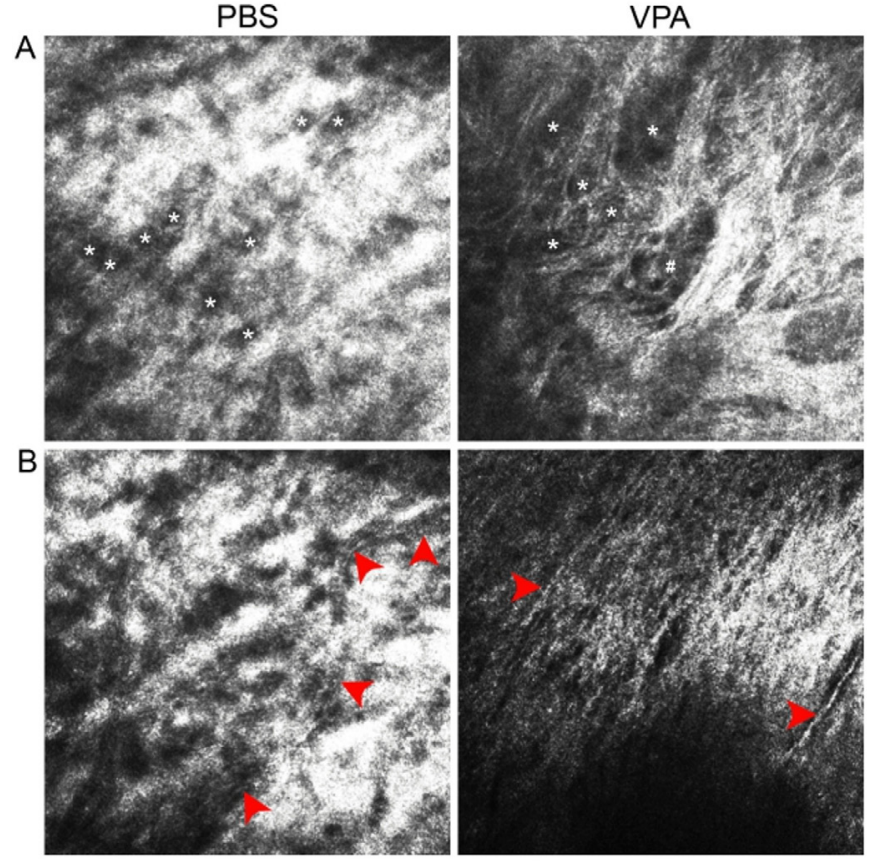

Figure 3 In vivo confocal microscopy of the day 28 operated conjunctivas in the rabbit model of minimally invasive glaucoma surgery. The same eyes examined by slit lamp in figure 2 were also imaged by confocal microscopy on day 28. (A) Visualisation of matrix organisationand cyst characteristics. Unencapsulated cysts are marked by ${ }^{*}$, while an example of an encapsulated cyst is marked by \#. (B) Visualisation of the stromal vasculature in the PBS-treated tissue; the course of a tortuous vessel was marked by the series of red arrowheads. In the VPA-treated tissue, two visible fine vessels were indicated by separate red arrowheads. PBS, phosphate buffered saline; VPA, valproic acid.

stromal meshwork of fibres was peppered with numerous cysts which were larger in size, and predominantly unencapsulated (figure 3A). With regard to the vasculature, blood vessels may be detected coursing through the hyper-reflective matrix in a tortuous manner in the PBS-treated tissue (figure 3B). In contrast, blood vessels detected in the VPA-treated bleb area appeared to be mainly straight (figure $3 \mathrm{~B}$ ). These observations suggest that VPA may enhance bleb function by promoting a bleb stroma consisting of looser connective tissue, larger-sized cysts and less tortuous vascular structure.

\section{Bleb extracellular matrix (ECM) organisation by histochemical evaluation}

We further examined the effect of VPA on collagen architecture in the operated conjunctiva by immunohistochemical analyses. Under the light microscope, operated tissue cryosections stained with $\mathrm{H} \& \mathrm{E}$, which highlighted the ECM in pink, confirmed that there were fundamental alterations in the VPA-treated ECM compared with PBS control. Whereas the PBS-treated bleb featured thick and disorganised ECM, the VPA-treated bleb is characterised by thinner and relatively more organised array of ECM fibres (figure 4A).

Next, we visualised the collagen matrix using Masson's trichrome staining, which uses three dyes to identify multiple tissue structures. Weigert's iron haematoxylin, Biebrich scarlet and aniline blue, applied sequentially, allow nuclei (black), cell cytoplasm (red) and collagen fibres (blue) to be distinguished, respectively. ${ }^{16}$ This method, although not specific to fibrillar 


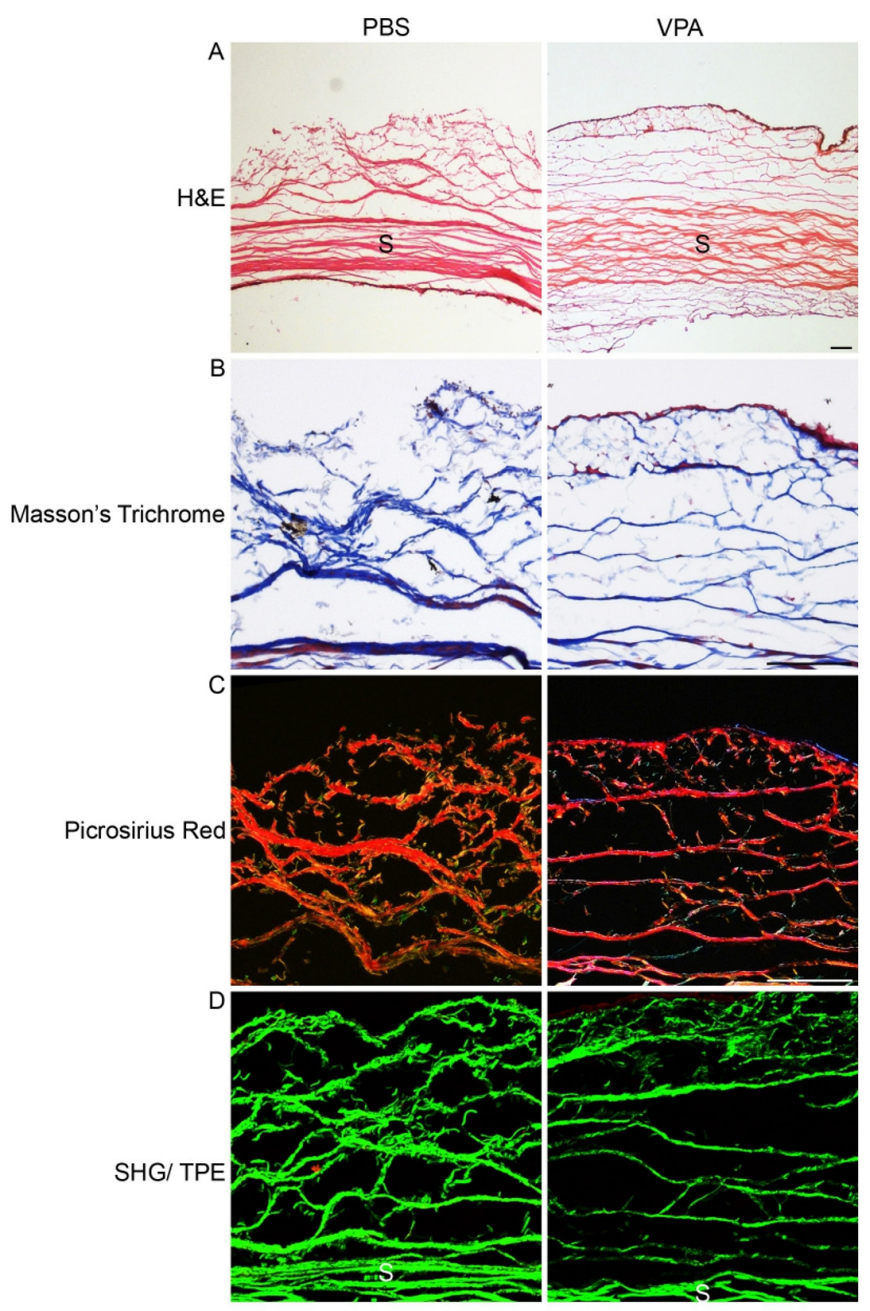

Figure 4 Histochemical and multiphoton visualisation of collagen characteristics in the rabbit model of minimally invasive glaucoma surgery treated with VPA. Serial frozen sections of the day 28 postoperative conjunctiva treated with either PBS or VPA were visualised via the indicated microscopy methods. The conjunctival cryosections were stained with (A) H\&E, (B) Masson's trichrome or (C) picrosirius red. The cryosections were stained with H\&E and Masson's trichrome $(A, B)$ were viewed under light microscopy, while the cryosection stained with picrosirius red $(C)$ was viewed via polarised microscopy. The stain-free cryosections were also viewed using multiphoton microscopy where the distribution of collagen fibres was revealed by green SHG signals. Scale bar $=100 \mu \mathrm{m}$. PBS, phosphate buffered saline; S, sclera; SHG, second harmonic generation; TPE, twophoton excitation; VPA, valproic acid.

collagen, is a commonly used collagen staining method for detection of fibrosis. Strikingly, similar pathologies were revealed by the trichrome stain, which showed abundant, thick collagen fibre deposition labelled in blue in the PBS-treated tissue, versus the predominantly fine collagen fibres present in the VPA-treated tissue (figure 4B).

Picrosirius red staining has been shown to be highly specific for fibrillar collagens when imaged under circularly polarised light. ${ }^{17}$ Considered as one of the gold standards in collagen imaging, this method reveals collagen bundles as red, orange, yellow or green fibres against a black background. ${ }^{17}$ Using this method, we again detected the pervasiveness of the thick and disorganised collagen fibre bundles in the PBS-treated tissue, as opposed to the conspicuously thinner collagen fibres in the VPAtreated bleb (figure 4C).

We further examined the collagen matrix using label-free multiphoton microscopy, which is also considered a gold standard for imaging collagen in tissues. ${ }^{18} 19$ This method, which is not limited by variability in staining protocols, clearly revealed the stark contrast in collagen fiber characteristics between the two treatment conditions (figure 4D). The SHG signals reiterated the aforementioned histochemical detections with high fidelity, confirming the induction of thick collagen fibres in the PBS-treated tissue versus the fine collagen fibres in the VPAtreated bleb.

\section{Collagen and FN colocalisation, apoptosis and cell growth by immunofluorescent evaluation}

We verified the differential collagen structures due to VPA treatment by immunofluorescent staining of the conjunctival cryosections with antibodies specific for type I collagen (COL1A1). The development of thicker collagen fibres in the PBS-treated conjunctiva and the permeation of the VPA-treated bleb by thinner and more diffusely distributed collagen fibres were confirmed (figure 5A). Coimmunofluorescence analysis with antibodies specific to $\mathrm{FN}$, another key fibrosis-associated protein, ${ }^{20}$ revealed FN to be closely associated with COL1A1. In fact, FN colocalised with majority of the collagen fibres and were also conspicuously thinner in the VPA-treated bleb compared with the PBS-treated counterpart (figure 5A).

To ascertain whether the VPA treatment regime caused undue cytotoxicity above control, we compared the VPA-treated tissues with PBS-treated counterparts for signs of apoptosis and cell proliferation. To detect apoptotic cells undergoing DNA degradation, we examined the conjunctival cryosections by TUNEL assay. Surprisingly, both PBS-treated and VPA-treated blebs featured prominent TUNEL positivity in the conjunctival epithelium, with much fewer TUNEL-positive cells in the stroma and episclera (figure 5B). Otherwise, there was no apparent difference in the level of apoptosis between the PBS control and VPA-treated tissue. The capacity for cellular regeneration in the day 28 operated tissues was visualised by immunostaining the cryosections with antibodies against Ki67, a cellular marker for proliferation. Conspicuous numbers of Ki67-positive cells were detected underneath the epithelium, with some colocalisation with TUNEL-positive cells occurring in the epithelium and stroma in both PBS-treated and VPA-treated tissues (figure 5B). These observations indicate that VPA did not suppress cell proliferation and suggest that the capacity for cellular recovery is not disrupted as cell proliferation coexisted with cell death in the treated tissues, a phenomenon similarly observed in the PBS control.

Taken together, the day 28 postoperative conjunctival pathologies were consistent with VPA being able to suppress the formation of thick and disorganised collagen/FN fibre bundles which are characteristic of the PBS-treated operated conjunctiva. This VPA effect occurred in a tissue background consisting of cells undergoing apoptosis and cell proliferation that similarly existed in the PBS-treated control.

\section{Fibrotic gene expression in the rabbit model of MIGS}

Reduced collagen and FN distribution in the VPA-treated bleb suggests that collagen and FN expression may be suppressed by VPA. We therefore proceeded to determine whether the quantity of collagen and FN transcripts in the operated rabbit conjunctivas were modulated by VPA. Indeed, the expression of both 

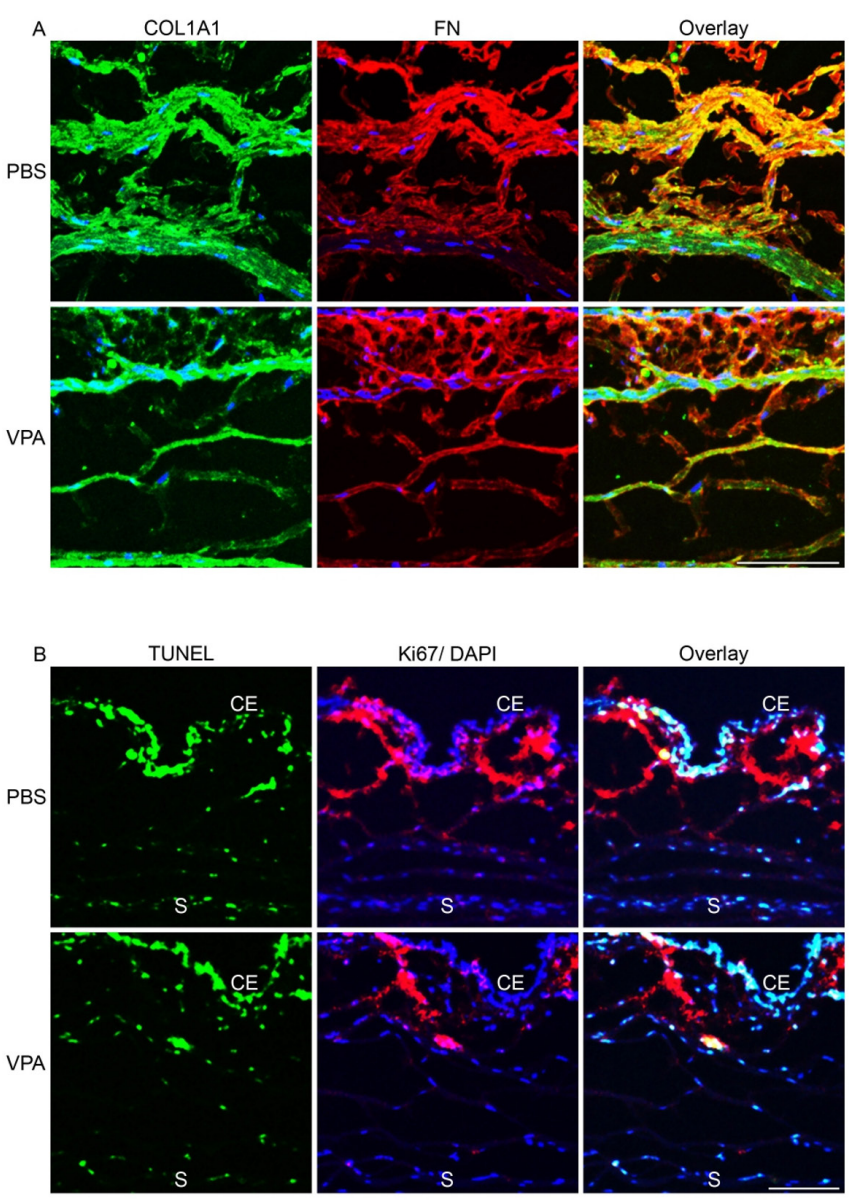

Figure 5 Immunofluorescent visualisation of collagen and FN characteristics, apoptosis and cell proliferation in the rabbit model of minimally invasive glaucoma surgery. (A) Serial cryosections of the same eyes examined in figure 4 were colabelled with antibodies specific for COL1A1 and FN. (B) Serial cryosections of the same eyes were colabelled with fluorescein-12-dUTP (green) as marker for apoptosis and antibody specific for Ki67 (red) as marker for proliferation. Nuclei were visualised by DAPI staining (blue). Images were taken by confocal microscopy. Scale bar $=100 \mu \mathrm{m}$. CE, conjunctival epithelium; FN, fibronectin; PBS, phosphate buffered saline; S, sclera; TUNEL, terminal deoxynucleotidyl transferase dUTP nick-end labelling; VPA, valproic acid.

Col1a1 and Fn genes in the day 28 rabbit tissues were significantly reduced by 2.02 -fold and 1.51 -fold, respectively, on VPA treatment when compared with PBS controls (figure 6). As Smad6 induction was implicated in the inhibition of Col1a1 by VPA in the mouse model of conjunctival scarring, ${ }^{21}$ we further probed whether Smad6 expression was also modulated in the rabbit model. Smad6 mRNA was significantly induced by 1.11 fold with VPA treatment, corroborating the previous finding in the mouse model. Hence, VPA was consistent in its capacity to modulate fibrotic changes in the ECM of the postoperative conjunctiva.

\section{DISCUSSION}

We show for the first time that VPA was effective in promoting a postoperative bleb morphology known to be associated with good bleb function in a rabbit model of MIGS. We further demonstrate that this effect may be attributed to the capacity of VPA to reduce the expression of key fibrosis-associated proteins
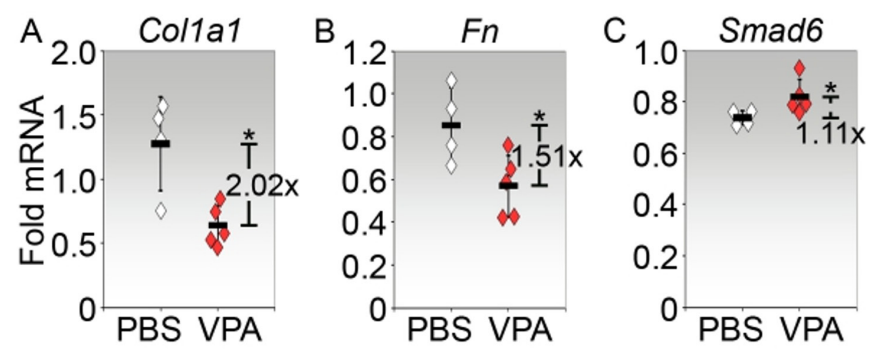

Figure 6 Gene expression in the rabbit model of minimally invasive glaucoma surgery treated with VPA. Rabbit conjunctiva tissues from both contralateral unoperated and implanted eyes were harvested on day 28 postsurgery and were subjected to analyses by real-time PCR for (A) Col1a1, (B) Fn and (C) Smad6. Values shown were calculated as fold changes from contralateral unoperated levels. Each symbol represents one rabbit eye ( $P B S, n=4 ; V P A, n=5$ ). The mean fold change $\pm S D$ for each condition is indicated by the horizontal bars. The fold difference in expression calculated as a ratio between the means of PBS and VPA expression is also indicated. ${ }^{*} \mathrm{P}<0.05$. PBS, phosphate buffered saline; VPA, valproic acid.

type I collagen and FN, and involved disruption of the TGF- $\beta$ signalling pathway.

The conjunctiva contains a collagen network that provides structure and strength to its role as a physical protective barrier against foreign material and infection. This ECM network is supported by fibroblasts residing within the stromal matrix, which remains quiescent when the tissue is undisturbed. When injured, such as in GFS, these fibroblasts are activated and have the capacity to transform the ECM network into one that consists of dense and disorganised collagen, the main mechanism for GFS failure. ${ }^{22} 23$ To determine whether VPA was able to modulate the fibrotic ECM, we have evaluated the postoperative conjunctival stromal organisation by various techniques, namely, live imaging and immunohistochemical analyses.

Non-invasive imaging techniques by slit-lamp biomicroscopy and in vivo confocal microscopy are well-established clinical assessment tools for postoperative bleb morphology. ${ }^{24}$ Slit-lamp biomicroscopy provides rapid macroscopic visualisation of the surgical outcome. Several studies have reported on the structural and morphological features of a successful bleb that are discernible by the slit lamp, and these have led to the development of bleb grading systems to classify blebs and manage their progress. ${ }^{25-27}$ On the other hand, in vivo confocal imaging allows the microstructure of filtering blebs to be viewed and provides details on the internal structural pattern of successful blebs. Qualities of the subconjunctival scar organisation revealed by confocal microscopy that correlated well with bleb morphology and function include blood vessel characteristics, stromal tissue pattern and stromal cyst appearance. ${ }^{428} 29$ Together, these two imaging techniques have provided a robust combination of macroscopic and microscopic indicators of surgical success, including a diffuse bleb with low elevation, minimal vascularisation, absence of tortuous conjunctival vessels, evidence of stromal cysts without capsule and reticular stromal pattern. ${ }^{45-272930}$

In this study, the blebs in the rabbit model of MIGS, where visible, were diffuse and tended to extend posteriorly from the proximity of the distal end of the implant. Notably, the presence of blebs in the later time points, particularly day 28, may only be observed in tissues treated with VPA. Early scarring in PBS controls is consistent with findings observed by other groups using a rabbit model. ${ }^{31-33}$ Given the diffuse appearance and the fact that bleb assessments are relatively subjective, with the risk 
of interobserver and/or intraobserver variability, we have not performed bleb survival analyses. Instead, we focused on the evaluation of bleb qualities deemed to be associated with functional blebs.

Many previous studies have reported that unfavourable bleb development was characterised by increased vascularisation and tortuous vessels. ${ }^{25}{ }^{34-37}$ Hence, bleb vascularity is an important early parameter to assess failure. In our study, the blebs treated with VPA generally featured less vascularity when viewed by slit-lamp biomicroscopy, particularly with respect to blood vessels crossing laterally across the bleb area. Moreover, when comparing the thickest blood vessels between the two conditions, it is obvious that the vessels in the VPA-treated tissue were thinner than in the PBS control, particularly on day 28. Although corkscrew vessels were detectable in both PBS-treated and VPA-treated bleb by slit lamp, in vivo microscopy of the day 28 tissues revealed straight and finer-looking vessels in the VPA bleb compared with the tortuous vessels typically seen in the PBS control. The reduced vascularisation and tortuosity of the involved vessels in the day 28 VPA bleb, as seen by slit-lamp biomicroscopy and in vivo confocal microscopy, may be indicative of an effective filtering bleb at 4 weeks. The vessels with narrower diameter in the VPA bleb may further correlate with good bleb function. ${ }^{29}$

The stromal fibre organisation, invisible to slit-lamp biomicroscopy but visible by in vivo confocal microscopy, has been correlated with bleb function. Four stromal patterns that are distinguishable by in vivo confocal microscopy, namely, trabecular, reticular, compact/hyper-reflective and blurred, have been described. ${ }^{49} 35$ The trabecular pattern, associated mainly with MMC-treated blebs, ${ }^{29} 35$ was not observed in the blebs in this study. The compact/hyper-reflective stroma, characterised by very densely packed, hyper-reflective fibres, was observed in the PBS-treated tissue. As this pattern was typically associated with bleb failure, ${ }^{429}$ the likelihood of the PBS treatment resulting in a non-functioning bleb before day 28 corroborated the slit-lamp biomicroscopic image. A combination of reticular and blurred stromal patterns may be observed in the VPA-treated tissue. In patients, the reticular pattern was significantly correlated with good bleb function, ${ }^{429}$ while also reported to be common to the later postoperative tissue, regardless of the bleb function. ${ }^{35}$

As normal conjunctiva also appeared to be characterised by a reticular stromal fibre arrangement, it was speculated that the reticular pattern may reflect the normalisation of superficial tissue architecture as wound healing process nears or achieves completion. ${ }^{35}$ Taking into account these considerations, our interpretation of the reticular stromal pattern associated with VPA treatment is that it prevents gross alteration in stromal organisation, and in so doing, maintains bleb function. On the other hand, the blurred stroma was reported to be typically present in the early first 2-3 months' postoperative tissue, and mainly associated with a reticular pattern. ${ }^{49}$ We therefore infer that VPA may delay the wound healing process so that the operated tissue takes on the appearance of the early postoperative period in the rabbit model, whereas obvious advanced scarring has already occurred in the PBS-treated tissue.

Besides the stromal fibre patterns, the stromal cystic spaces were also evaluated. While there is presently no agreement on whether encapsulated cysts in the stroma were correlated with bleb function, multiple studies have confirmed that the evidence of stromal cysts without capsules and a large total stromal cyst area correlated with good bleb function. ${ }^{429} 35$ In the VPA-treated blebs, both unencapsulated and encapsulated cystic spaces were observed concurrently. However, the most outstanding feature is the markedly larger size of the stromal cysts in the VPA-treated bleb compared with the mainly small cysts visualised in the PBStreated tissue. Overall, on the basis of these slit lamp and in vivo confocal images, we hypothesise that the evidence of reduced vascularisation and reticular/blurred stromal pattern with large stromal cysts in the VPA-treated bleb is indicative of aqueous outflow in a functional bleb.

Histological evaluations generally corroborate with in vivo confocal microscopy to suggest that a loosely arranged connective tissue is a key feature of functioning filtration blebs in patients. ${ }^{38-40}$ On the other hand, failed blebs are associated with extensive collagen production leading to a dense scar tissue obstructing the sclerostomy. ${ }^{38-40}$ Moreover, the failed scar matrix was described as containing many fibroblasts, much ground substance, parallel oriented collagen fibres, and contractile smooth muscle actin within the fibroblasts. ${ }^{41}$ In the present study, the major phenotype highlighted from immunohistochemical examinations is the presence of thick, disorganised collagen fibre bundles in the PBS-treated tissue matrix. The thick collagen fibres may be a reflection of high induction of collagen expression, as verified by the mRNA measurements. Fibre orientation, on the other hand, is an intriguing issue which may be related to the direction of movement of fibroblasts that synthesise them. ${ }^{42}{ }^{43}$ It is plausible therefore that there was a high degree of randomness in cell paths in the PBS-treated tissue in order to give rise to the apparent loss of fibre orientation. The implication then is that triggered fibroblasts in the tissue treated only with PBS may have behaved in a rather aggressive (high collagen expression) and chaotic (disorganised collagen assembly) fashion during wound healing. The existence of such a turbulent phase, on top of vascular damage caused by the surgery, may potentially lead to hypoxic foci and overexpression of proangiogenic factors, ${ }^{44} 45$ which then result in the development of disorganised, tortuous blood vessel networks observed, similar to those described in tumours. ${ }^{46}$ In contrast, the VPA-treated tissue matrix consisted of fine collagen fibres that appeared to much more aligned in parallel to the conjunctival surface. This collagen fibre phenotype, visible only in immunohistology and multiphoton microscopic examination, suggests that VPA may reduce the full impact of stimulation on fibroblasts. One of these stimuli may be TGF- $\beta 2$, whose profibrotic stimulation of collagen production by conjunctival fibroblasts may be blocked by VPA. ${ }^{11}$ We also confirmed that VPA upregulated Smad6, an inhibitor of the TGF- $\beta$ signalling pathway, ${ }^{47}$ in corroboration with our previous findings in a mouse model of conjunctival scarring. ${ }^{11}$ Another possible mechanism for the prevention of excessive collagen deposition by VPA may be the capacity of this drug to also cause reduction of FN expression. FN is well established to play a key role in collagen assembly, ${ }^{48} 49$ and its reduction by VPA may be expected to downgrade collagen assembly. Indeed, decreased FN deposition was apparent in the visibly thinner FN fibrils found in close association with the collagen network. As both collagen and FN are also major constituents of the blood vessel wall, ${ }^{50}$ the influence of VPA on collagen and FN expression on conjunctival stromal fibroblasts may extend to cells supporting the vasculature, thus explaining the thinner blood vessels observed. It is also possible that VPA may modulate the development of vascular abnormalities by reducing hypoxia through restraining the inflammatory response, which has a high metabolic demand for oxygen ${ }^{51}$ Overall, the distinct histological differences between PBS-treated and VPA-treated postoperative conjunctiva provide the evidence for the capacity of VPA to ameliorate the fibrotic phenotype, including vascular remodelling. 
Based on the apoptotic and cell proliferation profiles in the treated tissues, VPA used at the regime described did not cause any greater damage to the operated conjunctiva compared with treatment with PBS, nor did it suppress the capacity of the postoperative tissue to regenerate by cell proliferation. We suspect that the presence of apoptotic signals, which appeared particularly extensive in the conjunctival epithelium regardless of treatment condition, is likely due to physical disturbance caused by the multiple injections performed over the course of 3 weeks. However, any damage to the epithelium is expected to be short term, or may not occur at all, since proliferating cells, some of which were overlapping with apoptotic cells, were detected in the epithelium. Moreover, majority of the proliferating cells, likely fibroblasts, lurked in areas underlying the apoptotic epithelium. Given that stromal fibroblasts can directly influence the proliferation and differentiation of adjacent epithelial tissues through the provision of ECM and paracrine-acting growth factors and cytokines, any epithelial damage will likely be rapidly repaired.

MIGS are fast becoming ubiquitous, with safety and efficacy studies already being reported. ${ }^{52}{ }^{53}$ Despite the advent of these novel devices, the surgical technique continues to require the administration of cytotoxic MMC to mitigate fibrosis. With the present study demonstrating the potential capacity of VPA to support good bleb function without apparent cytotoxicity in a rabbit model of MIGS, the outlook is promising for the potential alternatives to MMC in the future.

Contributors ZLY designed the experiment, performed the animal surgery, clinical investigations before animal sacrifice, cleaning and analysis of some data, drafted and revised paper. L-FS harvested animal tissue after sacrifice, performed investigations, cleaned and analysed data, drafted and revised the paper. SWLC harvested animal tissue after sacrifice and performed investigations. LZT monitored data collection for the whole trial and assisted in animal handling. Fll assisted in animal handling and clinical investigations. TTW initiated the project, helped to design it and revised the paper.

Funding This work was supported by Singapore National Eye Centre Health Research Endowment Fund grant (HREF0218-3) and Clinician Scientist Award grant (NMRC/CSA-SI/0001/2015). Animal studies were partially funded by the SERI core grant (NMRC/CG/015/2013) administered by the Singapore Ministry of Health's National Medical Research Council. Santen Pharmaceutical Pte Ltd provided the PreserFlo implants.

\section{Competing interests None declared.}

\section{Patient consent for publication Not required.}

Ethics approval All experiments with animals were approved by the Institutional Animal Care and Use Committee and treated in accordance with the Association for Research in Vision and Ophthalmology Statement on the Use of Animals in Ophthalmic and Vision Research.

Provenance and peer review Not commissioned; externally peer reviewed.

Data availability statement Data are available upon reasonable request. Data are possibly available upon reasonable request depending on industry allowances.

Open access This is an open access article distributed in accordance with the Creative Commons Attribution Non Commercial (CC BY-NC 4.0) license, which permits others to distribute, remix, adapt, build upon this work non-commercially, and license their derivative works on different terms, provided the original work is properly cited, appropriate credit is given, any changes made indicated, and the use is non-commercial. See: http://creativecommons.org/licenses/by-nc/4.0/.

\section{ORCID iDs}

Zhu Li Yap http://orcid.org/0000-0002-4110-7539

Tina TWong http://orcid.org/0000-0003-1429-2895

\section{REFERENCES}

1 Heijl A, Leske MC, Bengtsson B, et al. Reduction of intraocular pressure and glaucoma progression: results from the early manifest glaucoma trial. Arch Ophthalmol 2002;120:1268-79.

2 Kerr NM, Wang J, Barton K. Minimally invasive glaucoma surgery as primary standalone surgery for glaucoma. Clin Exp Ophthalmol 2017;45:393-400.
3 Kerr J, Nelson P, O'Brien C. A comparison of ocular blood flow in untreated primary open-angle glaucoma and ocular hypertension. Am J Ophthalmol 1998;126:42-51.

4 Messmer EM, Zapp DM, Mackert MJ, et al. In vivo confocal microscopy of filtering blebs after trabeculectomy. Arch Ophthalmol 2006;124:1095-103.

5 Bell K, de Padua Soares Bezerra B, Mofokeng M, et al. Learning from the past: mitomycin C use in trabeculectomy and its application in bleb-forming minimally invasive glaucoma surgery. Surv Ophthalmol 2021;66:109-123.

6 Law SK, Modjtahedi SP, Mansury A, et al. Intermediate-Term comparison of trabeculectomy with intraoperative mitomycin-C between Asian American and Caucasian glaucoma patients: a case-controlled comparison. Eye 2007;21:71-8.

7 DeBry PW, Perkins TW, Heatley G, et al. Incidence of late-onset bleb-related complications following trabeculectomy with mitomycin. Arch Ophthalmol 2002;120:297-300.

8 Holló G, Healing W. Wound healing and glaucoma surgery: modulating the scarring process with conventional antimetabolites and new molecules. Dev Ophthalmol 2017;59:80-9.

9 Chateauvieux S, Morceau F, Dicato M, et al. Molecular and therapeutic potential and toxicity of valproic acid. J Biomed Biotechnol 201010.1155/2010/479364. [Epub ahead of print: 29 Jul 2010].

10 Khan S, Ahirwar K, Jena G. Anti-Fibrotic effects of valproic acid: role of HDAC inhibition and associated mechanisms. Epigenomics 2016;8:1087-101.

11 Seet L-F, Toh LZ, Finger SN, et al. Valproic acid suppresses collagen by selective regulation of Smads in conjunctival fibrosis. J Mol Med 2016;94:321-34.

12 Pinchuk L, Riss I, Batlle JF, et al. The use of poly(styrene-block-isobutylene-blockstyrene) as a microshunt to treat glaucoma. Regen Biomater 2016;3:137-42.

13 Seet L-F, Su R, Barathi VA, et al. SPARC deficiency results in improved surgical survival in a novel mouse model of glaucoma filtration surgery. PLoS One 2010;5:e9415.

14 Seet L-F, Finger SN, Chu SWL, et al. Novel insight into the inflammatory and cellular responses following experimental glaucoma surgery: a roadmap for inhibiting fibrosis. Curr Mol Med 2013;13:911-28.

15 Andersen CL, Jensen JL, Ørntoft TF. Normalization of real-time quantitative reverse transcription-PCR data: a model-based variance estimation approach to identify genes suited for normalization, applied to bladder and colon cancer data sets. Cancer Res 2004;64:5245-50

16 Sheehan DC HB. Theory and practice of Histotechnology. St. Louis: The Moxby Company, 1980.

17 Junqueira LC, Bignolas G, Brentani RR. Picrosirius staining plus polarization microscopy, a specific method for collagen detection in tissue sections. Histochem J 1979;11:447-55.

18 Zipfel WR, Williams RM, Christie R, et al. Live tissue intrinsic emission microscopy using multiphoton-excited native fluorescence and second harmonic generation. Proc Natl Acad Sci U S A 2003;100:7075-80.

19 Chen X, Nadiarynkh O, Plotnikov S, et al. Second harmonic generation microscopy for quantitative analysis of collagen fibrillar structure. Nat Protoc 2012;7:654-69.

20 Sible JC, Eriksson E, Smith SP, et al. Fibronectin gene expression differs in normal and abnormal human wound healing. Wound Repair Regen 1994;2:3-19.

21 Seet L-F, Narayanaswamy A, Finger SN, et al. Distinct iris gene expression profiles of primary angle closure glaucoma and primary open angle glaucoma and their interaction with ocular biometric parameters. Clin Exp Ophthalmol 2016;44:684-92.

22 Khaw PT, Occleston NL, Schultz G, et al. Activation and suppression of fibroblast function. Eye 1994;8 Pt 2:188-95.

23 Gurtner GC, Werner S, Barrandon Y, et al. Wound repair and regeneration. Nature 2008;453:314-21.

24 Ciancaglini M, Carpineto P, Agnifili L, et al. Filtering bleb functionality: a clinical, anterior segment optical coherence tomography and in vivo confocal microscopy study. J Glaucoma 2008;17:308-17.

25 Cantor LB, Mantravadi A, WuDunn D, et al. Morphologic classification of filtering blebs after glaucoma filtration surgery: the Indiana bleb appearance grading scale. $J$ Glaucoma 2003; 12:266-71.

26 Wells AP, Crowston JG, Marks J, et al. A pilot study of a system for grading of drainage blebs after glaucoma surgery. J Glaucoma 2004;13:454-60.

27 Wells AP, Ashraff NN, Hall RC, et al. Comparison of two clinical bleb grading systems. Ophthalmology 2006;113:77-83

28 Labbé A, Dupas B, Hamard P, et al. In vivo confocal microscopy study of blebs after filtering surgery. Ophthalmology 2005;112:1979

29 Caglar C, Karpuzoglu N, Batur M, et al. In vivo confocal microscopy and biomicroscopy of filtering blebs after trabeculectomy. J Glaucoma 2016;25:e377-83.

30 Picht G, Grehn F. Classification of filtering blebs in trabeculectomy: biomicroscopy and functionality. Curr Opin Ophthalmol 1998;9:2-8

31 Ma J, Li X, Zhang W, et al. CSM enhances the filtration bleb survival in rabbit model of experimental glaucoma surgery. Curr Eye Res 2014;39:982-8.

32 Lukowski ZL, Min J, Beattie AR, et al. Prevention of ocular scarring after glaucoma filtering surgery using the monoclonal antibody LT1009 (Sonepcizumab) in a rabbit model. J Glaucoma 2013;22:145-51.

33 Swogger J, Conner IP, Happ-Smith C, et al. Novel combination therapy reduces subconjunctival fibrosis after glaucoma filtration surgery in the rabbit model. Clin Exp Ophthalmol 2021;49:60-9. 
34 Sbeity Z, Radcliffe N, Palmiero P-M, et al. Non-contact in vivo scanning laser microscopy of blebitis. Eye 2009;23:1615-6.

35 Guthoff R, Klink T, Schlunck G, et al. In vivo confocal microscopy of failing and functioning filtering blebs: results and clinical correlations. J Glaucoma 2006;15:552-8.

36 Schwartz AL, Van Veldhuisen PC, Gaasterland DE, et al. The advanced glaucoma intervention study (AGIS): 5 . encapsulated bleb after initial trabeculectomy. Am J Ophthalmol 1999:127:8-19.

37 Sacu S, Rainer G, Findl O, et al. Correlation between the early morphological appearance of filtering blebs and outcome of trabeculectomy with mitomycin $C$.J Glaucoma 2003;12:430-5.

38 Addicks EM, Quigley HA, Green WR, et al. Histologic characteristics of filtering blebs in glaucomatous eyes. Arch Ophthalmol 1983;101:795-8.

39 Azuara-Blanco A, Katz LJ. Dysfunctional filtering blebs. Surv Ophthalmol 1998:43:93-126.

40 Filippopoulos T, Hanna E, Chen TC, et al. Correlation of filtration bleb morphology with histology. Int Ophthalmol Clin 2009;49:71-82.

41 Mietz H, Arnold G, Kirchhof B, et al. Histopathology of episcleral fibrosis after trabeculectomy with and without mitomycin C. Graefes Arch Clin Exp Ophthalmol 1996;234:364-8.

42 Laufer M, Ashkenazi C, Katz D, et al. Orientation of collagen in wound healing. $\mathrm{Br}$ J Exp Pathol 1974;55:233-6.

43 Cumming BD, McElwain DLS, Upton Z. A mathematical model of wound healing and subsequent scarring. J R Soc Interface 2010;7:19-34.
44 Chen L, Endler A, Shibasaki F. Hypoxia and angiogenesis: regulation of hypoxiainducible factors via novel binding factors. Exp Mol Med 2009;41:849-57.

45 Lokmic Z, Musyoka J, Hewitson TD, et al. Hypoxia and hypoxia signaling in tissue repair and fibrosis. Int Rev Cell Mol Biol 2012;296:139-85.

46 McDonald DM, Choyke PL. Imaging of angiogenesis: from microscope to clinic. Nat Med 2003:9:713-25.

47 Miyazawa K, Miyazono K. Regulation of TGF- $\beta$ family signaling by inhibitory Smads. Cold Spring Harb Perspect Biol 2017;9. doi:10.1101/cshperspect.a022095. [Epub ahead of print: 01 Mar 2017].

48 Kadler KE, Hill A, Canty-Laird EG. Collagen fibrillogenesis: fibronectin, integrins, and minor collagens as organizers and nucleators. Curr Opin Cell Biol 2008;20:495-501.

49 Paten JA, Martin CL, Wanis JT, et al. Molecular interactions between collagen and fibronectin: a reciprocal relationship that regulates de novo fibrillogenesis. Chem 2019:5:2126-45

$50 \mathrm{Xu} \mathrm{J,} \mathrm{Shi} \mathrm{G-P.} \mathrm{Vascular} \mathrm{wall} \mathrm{extracellular} \mathrm{matrix} \mathrm{proteins} \mathrm{and} \mathrm{vascular} \mathrm{diseases.}$ Biochim Biophys Acta 2014;1842:2106-19.

51 Seet L-F, Toh LZ, Finger SN, et al. Valproic acid exerts specific cellular and molecular anti-inflammatory effects in post-operative conjunctiva. J Mol Med 2019;97:63-75.

52 Green W, Lind JT, Sheybani A. Review of the Xen gel stent and InnFocus MicroShunt. Curr Opin Ophthalmol 2018;29:162-70.

53 Do AT, Parikh H, Panarelli JF. Subconjunctival microinvasive glaucoma surgeries: an update on the Xen gel stent and the PreserFlo MicroShunt. Curr Opin Ophthalmol 2020:31:132-8. 